\title{
Compostos bioactivos de papas para alimentação complementar
}

\section{Bioactive compounds in baby foods for complementary feeding}

\author{
Camila Pizzano Fonseca ${ }^{1}$, Fernanda A. Tamashiro ${ }^{2}$, Veridiana Vera \\ de Rosso $^{2}$, Patricia da Graça Leite Speridião ${ }^{3}$
}

\section{Resumo}

Objetivo: verificar a presença de compostos bioactivos em papas principais, recomendadas para alimentação complementar. Métodos: a amostra foi constituída por seis preparações de papas principais, escolhidas aleatoriamente, cujas receitas são encontradas em dois manuais de orientação alimentar infantil, veiculados no Brasil. Análise dos compostos bioactivos, com base nos compostos fenólicos totais, ácido ascórbico, carotenoides e a concentração de vitamina A pré-formada de cada papa, foi realizada. Resultados: os teores de compostos fenólicos variaram entre $25,8 \mathrm{mg}$ e $33,8 \mathrm{mg} / 100 \mathrm{~g}$. Os níveis de ácido ascórbico apresentaram valores entre $10,8 \mathrm{mg} / 100 \mathrm{~g}$ e 87,8 mg/100 g. Em relação ao conteúdo total de carotenoides, encontrou-se valores entre 7,4 $\mu \mathrm{g} / 100 \mathrm{~g}$ a 177,8 $\mu \mathrm{g} / 100 \mathrm{~g}$. Quanto à presença de vitamina $\mathrm{A}$, os valores obtidos variaram de 266 e $322 \mu \mathrm{g} / 100 \mathrm{~g}$. Conclusão: as papas principais propostas nesses manuais apresentam quantidade significativa de compostos bioactivos.

Palavras-chave: Alimentação complementar; Lactente; Antioxidantes.

\begin{abstract}
Objective: to verify the presence of bioactive compounds in baby foods recommended for complementary feeding. Methods: the sample consisted of six baby foods preparations, chosen at random, whose recipes are found in two manuals for infant food guidance, published in Brazil. Analysis of bioactive compounds, based on total phenolic compounds, ascorbic acid, carotenoids and the preformed vitamin A concentration of each baby foods were also evaluated. Results: the contents of phenolic compounds ranged from $25.8 \mathrm{mg}$ to $33.8 \mathrm{mg} / 100 \mathrm{~g}$. The levels of ascorbic acid showed values between $10.8 \mathrm{mg} / 100 \mathrm{~g}$ and $87.8 \mathrm{mg} / 100 \mathrm{~g}$. Regarding the total carotenoid content, values were found between $7.4 \mu \mathrm{g} / 100 \mathrm{~g}$ to $177.8 \mu \mathrm{g} / 100 \mathrm{~g}$. Regarding the presence of vitamin A, the values obtained ranged from 266 and $322 \mu \mathrm{g} / 100 \mathrm{~g}$. Conclusion: the baby foods proposed in these manuals, present a significant amount of bioactive compounds.
\end{abstract}

Keywords: Complementary feeding; Infant; Antioxidants.

${ }^{1}$ Graduação em Nutrição pela Universidade Federal de São Paulo (Unifesp), Santos, São Paulo, Brasil.

${ }^{2}$ Doutorado em Ciência de Alimentos pela Universidade Estadual de Campinas (Unicamp), Campinas, São Paulo, Brasil. Professoras Associadas III da Universidade Federal de São Paulo, Santos, São Paulo, Brasil.

${ }^{3}$ Doutorado em Nutrição pela Universidade Federal de São Paulo, São Paulo, São Paulo, Brasil. Professora Adjunta da Universidade Federal de São Paulo, Santos, São Paulo, Brasil.E-mail: patricia@dominustech.net 


\section{Introdução}

Alimentação complementar é definida por alimentação oferecida no período em que outros alimentos ou líquidos são oferecidos à criança, em adição ao leite materno. Esta deve oferecer quantidades suficientes de água, energia, proteínas, gorduras, vitaminas e minerais, por meio de alimentos seguros, que façam parte da cultura alimentar, sejam economicamente acessíveis, além de agradáveis à criança. ${ }^{(1)}$ Alimentos complementares, geralmente, são representados por cereais, frutas, legumes, verduras e tubérculos, além das carnes, gorduras e ovos. Os alimentos complementares, recomendados são os de alta densidade energética de alto conteúdo proteico e ricos em vitaminas e minerais. ${ }^{(2)}$ Destarte, se referem aos alimentos especialmente preparados para crianças pequenas, até que elas possam receber os alimentos consumidos pela família e, geralmente, são veiculados nas papas principais (almoço e jantar).

A prática alimentar inadequada nos dois primeiros anos de vida, principalmente entre as populações menos favorecidas, está intimamente associada ao aumento da morbidade representada pelas doenças infecciosas, desnutrição e carências específicas de micronutrientes, particularmente de ferro, zinco e vitamina A. ${ }^{(3)}$

Além dos macronutrientes, a ingestão de compostos bioactivos é essencial para o pleno desenvolvimento da criança, uma vez que atuam no fortalecimento do sistema imunológico e na prevenção de doenças crônico-degenerativas. Dentre os compostos bioactivos que devem ser consumidos em todas as faixas etárias, destacamos os carotenoides (principalmente os pró-vitamínicos A), compostos fenólicos e o ácido ascórbico. As frutas, verduras e legumes são especialmente ricas em compostos bioactivos que têm suas funções relacionadas ao seu potencial antioxidante, podendo atuar no combate às espécies reativas de oxigênio, estimulando o sistema imunológico, regulando a expressão gênica, além de desempenhar atividade antibacteriana e antiviral. ${ }^{(4-5)}$
Os carotenoides são compostos que apresentam coloração variando do amarelo ao vermelho. Além da atividade de pró-vitamina $\mathrm{A}$, os carotenoides desempenham outras funções biológicas relacionadas à capacidade antioxidante, atuando como sequestrador de radicais livres e desativador de oxigênio singlete. A presença de vitamina A no organismo humano é importante para o desempenho normal da visão, expressão gênica, desenvolvimento embrionário e da função imunológica. ${ }^{(6)}$ A vitamina A pode ser encontrada na forma de próvitamina $A$ (principalmente $\beta$-caroteno) em muitos vegetais, ou então, na forma de vitamina A préformada (retinol esterificado) em alimentos de origem animal, sendo que os alimentos com próvitamina $\mathrm{A}$, apresentam menor biodisponibilidade de vitamina A em relação aos alimentos com vitamina A pré-formada. ${ }^{(7)}$

O ácido ascórbico tem papel importante no organismo e está envolvido no metabolismo da tirosina, ácido fólico e triptofano, assim como contribui na síntese de carnitina e de catecolaminas que auxiliam na regulação do sistema nervoso. ${ }^{(5-6)}$ Assim, considerando que a introdução da alimentação complementar é uma etapa importante para o desenvolvimento infantil, mas também vulnerável às deficiências nutricionais que podem impactar a vida toda, nosso estudo objetivou avaliar a presença de compostos bioactivos nas papas principais recomendadas para lactentes de 6 a 12 meses de idade.

\section{Métodos}

A amostra foi constituída de papas principais, escolhidas aleatoriamente, cujas receitas são encontradas em dois manuais de orientação alimentar para crianças, utilizados no $\operatorname{Brasil}^{(6-7)}$ na época da realização do estudo. As papas incluíram os seguintes ingredientes: 1) cará, quiabo e frango; 2) mandioca, abobrinha e carne moída; 3 ) abóbora, carne e feijão; 4) abóbora, couve e carne; 5) batata, espinafre, cenoura e frango; 6) mandioca, brócolis, beterraba e fígado (Quadro 1). As preparações 
foram realizadas no Laboratório de Dietética do Curso de Nutrição da Universidade Federal de São Paulo (Unifesp) - Campus Baixada Santista, de acordo com as técnicas de preparo descritas nos manuais, e todas as amostras foram transferidas para sacos plásticos com fecho hermético e congeladas em freezer $-40{ }^{\circ} \mathrm{C}$ por um período de duas horas. Logo após o congelamento, as amostras foram liofilizadas e armazenadas até a realização das análises.

Quadro 1 - Ingredientes utilizados nas papas principais.

\begin{tabular}{|c|c|}
\hline Papa principal & Ingredientes \\
\hline 1) Cará, quiabo e frango & $\begin{array}{l}2 \text { colheres (sopa) de peito de frango, sem pele, picadinho; } 1 \text { colher (sobremesa) } \\
\text { de óleo de soja; } 1 \text { colher (chá) de cebola ralada; } 1 \text { cará médio ( } 150 \text { g); } 1 \text { colher } \\
\text { (sopa) de quiabo picadinho; } 1 \text { colher (sopa) de feijão cozido (grão e caldo); } \\
1 \text { colher (café) rasa de sal; } 2 \text { copos de água. }\end{array}$ \\
\hline $\begin{array}{l}\text { 2) Mandioca, abobrinha } \\
\text { e carne moída }\end{array}$ & $\begin{array}{l}2 \text { colheres (sopa) de carne de vaca, magra, moída; } 1 \text { colher (sobremesa) de } \\
\text { óleo de soja; } 1 \text { colher (chá) de cebola ralada; } 2 \text { pedaços médios de mandioca } \\
(140 \text { g); } 1 \text { abobrinha pequena; } 1 \text { folha de couve picadinha; } 1 \text { colher (café) rasa } \\
\text { de sal; } 2 \text { copos de água. }\end{array}$ \\
\hline $\begin{array}{l}\text { 3) Abóbora, carne } \\
\text { e feijão }\end{array}$ & $\begin{array}{l}2 \text { colheres (sopa) de carne de boi, moída; } 1 \text { colher (sopa) de feijão (grão e } \\
\text { caldo); } 2 \text { colheres (chá) de óleo de soja; } 1 \text { colher (chá) de cebola; } 1 / 2 \text { pedaço } \\
\text { de alho; } 1 \text { colher (chá) de pimentão verde; } 1 \text { fatia grande de abóbora/moranga; } \\
1 \text { colher (café) de sal; } 1 \text { e } 1 / 2 \text { copo de água. }\end{array}$ \\
\hline $\begin{array}{l}\text { 4) Abóbora, couve } \\
\text { e carne }\end{array}$ & $\begin{array}{l}1 \text { pedaço médio de jerimum }(150 \mathrm{~g}) ; 1 \text { colher (sopa) cheia de taioba picada } \\
\text { (ou outra folha verde-escura); } 2 \text { colheres (sopa) cheias de carne moída ( } 50 \mathrm{~g}) \text {; } \\
1 \text { colher (chá) de óleo; } 1 \text { colher (café) rasa de sal; } 1 \text { colher (chá) de cebola } \\
\text { ralada; } 1 \text { pitada de orégano. }\end{array}$ \\
\hline $\begin{array}{l}\text { 5) Batata, espinafre, } \\
\text { cenoura e frango }\end{array}$ & $\begin{array}{l}1 \text { batata média }(100 \mathrm{~g}) ; 1 / 2 \text { cenoura }(50 \mathrm{~g}) ; 1 \text { colher (sopa) cheia de espinafre } \\
\text { picado (ou outra folha verde-escura); } 2 \text { colheres (sopa) cheias de galinha } \\
\text { desfiada ou } 1 \text { coxa }(50 \mathrm{~g}) ; 1 \text { colher (pequena) de óleo; } 1 \text { colher (café) rasa de } \\
\text { sal; } 1 \text { colher (chá) de cebola ralada; } 1 \text { colher (chá) de tomate picado. }\end{array}$ \\
\hline $\begin{array}{l}\text { 6) Mandioca, brócolis, } \\
\text { beterraba e fígado }\end{array}$ & $\begin{array}{l}1 / 2 \text { mandioca média }(150 \mathrm{~g}) ; 2 \text { ramos de brócolis; } 2 \text { fatias de beterraba; } \\
2 \text { colheres (sopa) cheias de fígado picado }(50 \mathrm{~g}) ; 1 \text { colher (pequena) de óleo; } \\
1 \text { colher (café) rasa de sal; } 1 \text { colher (chá) de cebola ralada; } 1 \text { colher (chá) de } \\
\text { cheiro verde picado. }\end{array}$ \\
\hline
\end{tabular}

Fonte: Sociedade Brasileira de Pediatria ${ }^{(6)}$ e Ministério da Saúde.(7)

O teor de compostos fenólicos totais foi determinado pelo método de Folin-Ciocalteau segundo procedimento de Singleton \& Rossi. ${ }^{(8)} \mathrm{O}$ teor de ácido ascórbico foi determinado quantitativamente, através de reação com 2,6-diclorofenolindofenol, segundo o método padrão da $\mathrm{AOAC},{ }^{(9)}$ modificado por Benassi \& Antunes. ${ }^{(10)}$ Os teores de compostos fenólicos e ácido ascórbico foram expressos em mg/100 g.

O perfil de carotenoides foi analisado por cromatografia líquida de alto desempenho, com utilização do método proposto por De Rosso e Mercadante. ${ }^{(11)}$ A quantificação dos carotenoides foi realizada empregando-se curvas-padrão de, no mínimo, sete concentrações diferentes para o alltrans- $\beta$-caroteno, all-trans- $\beta$-criptoxantina e alltrans-luteína. O teor de vitamina A total foi obtido de acordo com o método do Instituto Adolfo Lutz (IAL). ${ }^{(12)}$ Os carotenoides foram expressos em $\mu \mathrm{g} / 100 \mathrm{~g}$. O estudo foi aprovado pelo Comitê de Ética em Pesquisa da Universidade Federal de São Paulo. 


\section{Resultados}

A concentração de compostos fenólicos totais das diferentes amostras de papas principais variou de 24,9 a $35,9 \mathrm{mg} / 100 \mathrm{~g}$, sendo que as papas
4 e 6 apresentaram os maiores valores. A concentração de ácido ascórbico apresentou grande variação, sendo encontrados $10,8 \mathrm{mg} / 100 \mathrm{~g}$ na papa 1 e $87,8 \mathrm{mg} / 100 \mathrm{~g}$ na papa 6 , conforme se observa na Tabela 1.

Tabela 1 - Médias e desvios-padrão de compostos fenólicos totais e ácido ascórbico das papas principais analisadas no estudo.

\begin{tabular}{lcc}
\hline Papas principais & $\begin{array}{c}\text { Compostos fenólicos totais } \\
(\mathbf{m g} / \mathbf{1 0 0} \mathbf{g})\end{array}$ & $\begin{array}{c}\text { Ácido ascórbico } \\
(\mathbf{m g} / \mathbf{1 0 0} \mathbf{~})\end{array}$ \\
\hline 1) Cará, quiabo e frango & $25,8 \pm 2,7$ & $10,8 \pm 1,0$ \\
2) Mandioca, abobrinha e carne moída & $28,9 \pm 0,1$ & $69,7 \pm 0,0$ \\
3) Abóbora, carne e feijão & $24,9 \pm 0,4$ & $22,9 \pm 0,0$ \\
4) Abóbora, couve e carne & $35,9^{*}$ & $35,2 \pm 2,1$ \\
5) Batata, espinafre, cenoura e frango & $26,2 \pm 1,6$ & $27,0 \pm 0,1$ \\
6) Mandioca, brócolis, beterraba e fígado & $33,9^{*}$ & $87,8 \pm 0,4$ \\
\hline
\end{tabular}

*Não se calculou a média \pm desvio-padrão, pois uma das amostras foi perdida durante a análise.

Fonte: as autoras.

Os carotenoides das papas principais e suas concentrações foram identificados pela presença de neoxantina; anteraxantina; luteína; zeaxantina; 5,6-epóxi- $\beta$-criptoxantina; 5,8-epóxi- $\beta$-caroteno; $\alpha$-criptoxantina; $\beta$-criptoxantina; $\alpha$-caroteno e $\beta$-caroteno (Tabela 2).

Tabela 2 - Concentração de carotenoides totais das papas principais analisadas no estudo.

\begin{tabular}{lcccccc}
\hline \multirow{2}{*}{ Carotenoides } & \multicolumn{7}{c}{ Concentração $(\boldsymbol{\mu g} / \mathbf{1 0 0}$ g) } \\
\cline { 2 - 7 } & Papa 1 & Papa 2 & Papa 3 & Papa 4 & Papa 5 & Papa 6 \\
\hline 9-cis-neoxantina & ND & ND & 9,9 & ND & ND & ND \\
all-trans-anteraxantina & ND & ND & ND & 3,5 & ND & ND \\
all-trans-luteína & 1,1 & 1,2 & 52,7 & 14,7 & 8,7 & 0,7 \\
all-trans-zeaxantina & 0,3 & ND & ND & 3,7 & ND & ND \\
5,6-epoxi- $\beta$-criptoxantina & ND & ND & ND & ND & 5,1 & 15,5 \\
all-trans- $\alpha$-criptoxantina & ND & ND & 62,2 & ND & ND & 3,7 \\
5,8-epoxi- $\beta$-caroteno & ND & ND & ND & 6,0 & ND & ND \\
all-trans- $\beta$-criptoxantina & ND & ND & 19,4 & ND & 6,4 & 18,4 \\
13-cis- $\beta$-caroteno & ND & ND & ND & 5,4 & ND & ND \\
all-trans- $\alpha$-caroteno & ND & ND & ND & ND & 7,6 & ND \\
all-trans- $\beta$-caroteno & 5,1 & 3,2 & 28,8 & 54,0 & 9,1 & 13,6 \\
9-cis- $\beta$-caroteno & 3,6 & 2,9 & 4,5 & 6,5 & 3,3 & 3,7 \\
Carotenoides totais & 10,3 & 7,4 & 177,8 & 94,1 & 40,6 & 55,8 \\
Pró-vitamina A (RAE) & 0,5 & 0,3 & 5,9 & 5,2 & 1,7 & 2,8 \\
\hline
\end{tabular}

Legenda: ND: não detectado; papa 1 (cará, quiabo e frango); papa 2 (mandioca, abobrinha e carne moída); papa 3 (abóbora, carne e feijão); papa 4 (abóbora, couve e carne); papa 5 (batata, espinafre, cenoura e frango); papa 6 (mandioca, brócolis, beterraba e fígado).

Fonte: as autoras. 
Verifica-se na Tabela 2 que os teores de carotenoides totais variaram entre $7,4 \mu \mathrm{g} / 100 \mathrm{~g}$ na papa 2 e $177,8 \mu \mathrm{g} / 100 \mathrm{~g}$ na papa 3 , sendo que o maior teor de all-trans- $\beta$-caroteno foi de $54,0 \mu \mathrm{g} / 100 \mathrm{~g}$, encontrado na papa 4.
A seguir, observa-se que a concentração de vitamina A pré-formada variou de $266 \mu \mathrm{g}$ na papa 2 a $1322 \mu \mathrm{g} / 100 \mathrm{~g}$ na papa 6 . Os teores de vitamina A total mostraram resultados entre $266,4 \mu \mathrm{g} / 100 \mathrm{~g}$ na papa 2 e $1323,7 \mu \mathrm{g} / 100 \mathrm{~g}$ na papa 6 (Tabela 3 ).

Tabela 3 - Concentração de vitamina A pré-formada e total das papas principais analisadas no estudo.

\begin{tabular}{lcc}
\hline Papas principais & $\begin{array}{c}\text { Vitamina A pré-formada } \\
(\boldsymbol{\mu g} / \mathbf{1 0 0} \mathbf{g})\end{array}$ & $\begin{array}{c}\text { Vitamina A total } \\
(\boldsymbol{\mu g} / \mathbf{1 0 0} \mathbf{g})\end{array}$ \\
\hline 1) Cará, quiabo e frango & 283 & 289,0 \\
2) Mandioca, abobrinha e carne moída & 266 & 266,4 \\
3) Abóbora, carne e feijão & 511 & 516,2 \\
4) Abóbora, couve e carne & 314 & 316,9 \\
5) Batata, espinafre, cenoura e frango & 336 & 336,6 \\
6) Mandioca, brócolis, beterraba e fígado & 1322 & 1323,7 \\
\hline
\end{tabular}

Fonte: as autoras.

\section{Discussão}

A partir dos seis meses de idade as necessidades nutricionais do lactente não mais são supridas exclusivamente pelo aleitamento materno, sendo necessária introdução da alimentação complementar para atender às demandas de energia, proteínas, vitaminas e minerais. ${ }^{(13)}$

No território nacional, estudos avaliaram a alimentação complementar de lactentes com base na ingestão alimentar e práticas alimentares de crianças nos primeiros anos de vida, ${ }^{(14-18)}$ mostrando inadequação de macro e micronutrientes, além de evidenciar baixa densidade energética nas refeições dessas crianças.

Nosso estudo apontou a presença de compostos bioactivos, caracterizando-se como um referencial na prática de profissionais envolvidos com a assistência pediátrica. Compostos bioactivos apresentam grande potencial antioxidante e atuam no combate às espécies reativas de oxigênio, estimulando o sistema imunológico, regulando a expressão gênica, além de desempenhar atividade antibacteriana e antiviral. ${ }^{(4-5)}$

Os maiores teores de compostos fenólicos foram encontrados nas papas principais de número
4 (abóbora, couve e carne) e 6 (mandioca, brócolis, beterraba e fígado), possivelmente pela presença de couve em suas preparações. Estudo realizado em Recife, Pernambuco, no qual foi determinada a concentração de compostos fenólicos em frutas e hortaliças, também mostrou que a couve é rica nesses compostos bioactivos quando comparada às outras hortaliças. ${ }^{(19)}$

Já o ácido ascórbico apresentou maior concentração na papa 6 , o que pode ser devido à presença do brócolis, considerado um alimento rico nesse nutriente. ${ }^{(20)}$ Nas papas 3 e 5 é possível observar que a concentração de ácido ascórbico apresentou-se em menor quantidade, permitindo sugerir que a presença do feijão e do espinafre promoveram menor retenção desse nutriente durante o cozimento. ${ }^{(21)}$

Quanto aos teores de carotenoides totais, as papas 3 e 4 apresentaram os maiores valores, provavelmente pela presença de abóbora nas suas composições. A abóbora (Cucurbita moschata), nativa das Américas, apresenta altos teores de carotenos. ${ }^{(22)}$ Em relação à vitamina $\mathrm{A}$ pode-se dizer que os teores de $\beta$-caroteno contribuíram, predominantemente, com a concentração de vitamina A pré-formada e $100 \%$ desta foi convertida em 
vitamina A total. Esses resultados podem ser respaldados pelas análises das papas 3 e 6 , cujas preparações contaram com a presença da abóbora e do fígado bovino, considerados excelentes fontes de vitamina $\mathrm{A} .{ }^{(22-23)}$

É possível sugerir que nosso estudo é bastante relevante e pioneiro, pois permitiu avaliar a presença de compostos bioactivos em papas principais propostas em dois manuais brasileiros de recomendação para alimentação complementar. Entretanto, é importante ressaltar que o presente estudo apresenta limitação, pois nosso universo amostral foi pequeno; contudo, possibilitou a verificação da presença valiosa dos compostos bioactivos. Outro aspecto que deve ser destacado é o fato de que a discussão dos nossos resultados se baseou na literatura brasileira, dada dificuldade de comparação de dados com outros alimentos utilizados em culturas alimentares distintas da nossa, além da diferente composição nutricional.

Assim, diante dos resultados encontrados é possível concluir que as papas principais avaliadas contam com a presença de compostos bioactivos necessários à alimentação complementar de crianças.

\section{Agradecimentos}

As autoras agradecem à Fundação de Amparo à Pesquisa do Estado de São Paulo - FAPESP (Processo 2011/11623-3 e Processo 2009/53884-8) pelo apoio financeiro a esta pesquisa.

\section{Conflito de interesses}

As autoras declaram não haver conflito de interesses, seja financeiro ou outro, direto e/ou indireto.

\section{Referências}

1 Speridião PGL. Alimentação complementar. Nutr. \& Pediat. 2010; 2(1):10-3.

2 Silveira FJF, Lamounier JA. Prevalência do aleitamento materno e práticas de alimentação complementar em crianças com até 24 meses de idade na região do Alto Jequitinhonha, Minas Gerais. Rev Nutr. 2004;17(4):437-47. doi: $10.1590 / \mathrm{S} 1415-52732004000400004$

3 Monte CMG, Giugliani ERJ. Recomendações para alimentação complementar da criança em aleitamento materno. J Pediatr. 2004,80(5):S 131-41. doi: 10.1590/S0021-7557200400070 0004

4 Szajdek A, Borowska EJ. Bioactive compounds and health-promoting properties of berry fruits: a review. Plant Foods Hum Nutr. 2008;63:147-56. doi: 10.1007/s11130-0080097-5

5 Leong LP, Shui G. An investigation of antioxidant capacity of fruits in Singapore markets. Food Chem. 2002;76:69-75. doi: 10.1016/ S0308-8146(01)00251-5

6 Sociedade Brasileira de Pediatria. Manual de orientação para a alimentação do lactente, do pré-escolar, do escolar, do adolescente e na escola. [Internet]. Rio de Janeiro: Sociedade Brasileira de Pediatria; 2008 [citado 2018 jun 10]. Disponível em: https://www.sbp.com. br/fileadmin/user_upload/pdfs/10478e-Man Nutrologia.pdf

7 Brasil. Ministério da Saúde. Saúde da Criança: Nutrição Infantil - Aleitamento Materno e Alimentação Complementar. [Internet]. Brasília: Ministério da Saúde; 2015 [citado 2018 jun 23]. Disponível em: https://bvsms.saude.gov.br/ bvs/publicacoes/saude_crianca_aleitamento_ materno_cab23.pdf

8 Singleton VL, Rossi JA. Colorimetry of total phenolics with phosphomolybdic-phosphotungstic acid reagents. Am J Enol Viticult. 1965;16:144-58.

9 Association of Official Analitycal Chemists. Official methods of analysis. Washington: AOAC; 1984.

10 Benassi MT, Antunes AJ. A comparison of metaphosphoric and oxalic acids as extractans solutions for the determination of vitamin $\mathrm{C}$ in selected vegetables. Arq Biol Technol. 1988; 31(4):507-13. 
11 Rosso VV, Mercadante AZ. Identification and quantification of carotenoids by HPLCPDA-MSn from amazonian fruits. J Agric Food Chem. 2007;55:5062-72. doi: 10.1021/ jf0705421

12 Instituto Adolfo Lutz. Métodos físico-químicos para análise de alimentos. São Paulo: Instituto Adolfo Lutz; 2008.

13 Dias MCAP, Freire LMS, Franceschini SCC. Recomendações para alimentação complementar de crianças menores de dois anos. Rev Nutr. 2010;23(3):475-86.

14 Marchioni DM, Latorre MR, Szarfac SC, Souza SB. Complementary feeding: study on prevalence of food intake in two health centers of São Paulo city. Arch Latinoam Nutr. 2001; 51(2):161-6.

15 Oliveira LPM, Assis AMO, Pinheiro SMC, Prado MS, Barreto ML. Alimentação complementar nos primeiros dois anos de vida. Rev Nutr. 2005;18(4):459-69. doi: 10.1590/S141552732005000400002

16 Ribeiro P. Composição química de preparações culinárias salgadas destinadas a crianças menores de 18 meses de idade [tese]. São Paulo: Universidade Federal de São Paulo; 2006.

17 Saldiva SRDM, Escuder MM, Mondini L, Levy RB, Venancio SI. Práticas alimentares de crianças de 6 a 12 meses e fatores maternos associados. J Pediatr. 2007;83(1):53-8.

18 Portella MB, Morais TB, Morais MB. Excesso de sódio e déficit de ferro em alimentos de transição. J Pediatr. 2010;86(4):303-10. doi: 10.1590/S0021-75572010000400010

19 Mélo EA, Lima VLG, Maciel MIS. Polyphenol, ascorbic acid and total carotenoid contents in commom fruits and vegetables. Brazilian Journal of Food Technology. 2006;9(2):89-94.

20 Giugliani ERJ, Victoria CG. Alimentação complementar. J.Pediatr.2000;76(Supl.3):S253-62.

21 Cury RDB. Compostos bioativos presentes em produtos infantis e sua ação anti-radical livre. [tese]. Campinas (SP): Universidade Estadual de Campinas; 2008.
22 Souza CO, Menezes JDS, Ramos Neto DC, Assis JCA, Silva SR, Druzian JI. Carotenoides totais e vitamina A de cucurbitáceas do Banco Ativo de Germoplasma da Embrapa Semiárido. Ciênc. Rural. 2012;42(5). Doi: 10.1590/ S0103-84782012005000024

23 Coultate TP. Alimentos: a química de seus componentes. Porto Alegre: Artmed; 2004.
Recebido em: 27 out. 2020

Aceito em: 7 jan. 2021 
Fonseca, C. P. et al. 